\title{
Estimating Surgical Outcomes for Hepatocellular Carcinoma
}

\author{
Curtis J. Wray, MD \\ Department of Surgery, University of Texas Health Science Center at Houston, Houston, TX
}

Hepatocellular carcinoma (HCC) remains a problematic, common gastrointestinal malignancy. Short- and long-term outcomes are suboptimal as the cancer and underlying cirrhosis present two significant clinical challenges. Just with the presence of cirrhosis, patients have a chronic illness with a median survival that ranges from 2 years in decompensated cases up to 12 years in those with compensated liver disease. ${ }^{1}$ The addition of malignancy to the cirrhosis presents a lethal combination and the worldwide burden of HCC is estimated to exceed 1 million deaths by $2030 .^{2}$ In the United States, the HCC mortality rate increased by $43 \%$ from 2000 to 2016 with 5 -year survival only marginally better than pancreas cancer. ${ }^{3}$ These grim statistics highlight a major knowledge gap in every facet related to the diagnosis and treatment of liver cancer.

Because liver transplantation and organ allocation are supply limited, surgical resection is recommended for those with minimal cirrhosis, portal hypertension, and suitable candidates (small lesions $<3 \mathrm{~cm}$ ). ${ }^{4}$ Liver resection for HCC has evolved significantly over the past 20 years, making it a much safer procedure when performed in the proper context with adequate patient selection. ${ }^{5}$ Cirrhosis is a wellknown risk factor for serious postoperative complications, including hemorrhage, bile leak, post-hepatectomy liver failure, and death. Improved understanding and preoperative risk estimation is critical to minimize postoperative complications. Various strategies have been studied to help guide liver resection and to improve long-term outcomes. ${ }^{6}$ In the report by Haruki et al., ${ }^{7}$ the authors investigate the association between rapid turnover proteins and HCC surgical outcomes. The rapid protein turnover score $>2$ was significantly associated with worse survival.

(C) Society of Surgical Oncology 2021

First Received: 16 August 2021

Accepted: 8 September 2021;

Published Online: 22 September 2021

C. J. Wray, MD

e-mail: curtis.j.wray@uth.tmc.edu
These data and conclusions must be interpreted carefully. Validation in a larger, external data set is needed before widespread adoption. However, at present this paper provides hypothesis-generating ideas, and the use of this novel, noninvasive scoring system may aid clinicians in the preoperative estimation of surgical risk. The nutritional impact of advanced cirrhosis and HCC has long been established as ascites, hypoalbuminemia, and cachexia negatively impact quality of life. For those patients with marginal cirrhosis, use of this type of scoring system may help to identify those patients at-risk for poor outcomes and/or complication. The rapid turnover protein score may prove useful once confirmed as a predictor of surgical outcome following resection of hepatocellular carcinoma.

\section{REFERENCES}

1. D'Amico G, Garcia-Tsao G, Pagliaro L. Natural history and prognostic indicators of survival in cirrhosis: a systematic review of 118 studies. J Hepatol. 2006;44(1):217-31.

2. Villanueva A. Hepatocellular carcinoma. $N$ Engl $J$ Med. 2019;380(15):1450-62.

3. Jemal A, Ward EM, Johnson CJ, et al. Annual report to the nation on the status of cancer, 1975-2014, featuring survival. J Natl Cancer Inst. 2017. https://doi.org/10.1093/jnci/djx030.

4. Orcutt ST, Anaya DA. Liver resection and surgical strategies for management of primary liver cancer. Cancer Control. 2018;25(1):1073274817744621.

5. Benson AB, D'Angelica MI, Abbott DE, et al. Guidelines insights: hepatobiliary cancers, version 2.2019. J Natl Compr Cancer Network. 2019;17(4):302-10.

6. Allenson K, Roife D, Kao LS, Ko TC, Wray CJ. Estimation of hepatocellular carcinoma mortality using aspartate aminotransferase to platelet ratio index. $J$ Gastrointest Oncol. 2020;11(2):291-7.

7. Yanagaki M, Haruki K, Yasuda J, et al. The significance of the rapid turnover protein score as a predictor of the long-term outcomes in hepatocellular carcinoma after hepatic resection. Ann Surg Oncol. 2021. https://doi.org/10.1245/s10434-021-10704-9.

Publisher's Note Springer Nature remains neutral with regard to jurisdictional claims in published maps and institutional affiliations. 